\title{
Three-incision robotic major lung resection for cancer
}

\section{Kazuhiro Ueda, Tadashi Umehara, Koki Maeda, Soichi Suzuki, Naoya Yokomakura, Kota Kariatsumari, Masami Sato}

Department of General Thoracic Surgery, Kagoshima University Graduate School of Dental and Medical Sciences, Kagoshima, Japan

Contributions: (I) Conception and design: K Ueda; (II) Administrative support: None; (III) Provision of study materials or patients: None; (IV)

Collection and assembly of data: K Ueda, T Umehara, S Suzuki, N Yokomakura, K Kariatsumari, M Sato; (V) Data analysis and interpretation: K Ueda, M Sato; (VI) Manuscript writing: All authors; (VII) Final approval of manuscript: All authors.

Correspondence to: Kazuhiro Ueda, MD, PhD. Department of General Thoracic Surgery, Kagoshima University Graduate School of Medical and Dental Sciences, 8-35-1 Sakuragaoka, Kagoshima 890-8520, Japan. Email: k7433286@kadai.jp.

\begin{abstract}
Background: Regardless of the current trend in reduced port surgery, robotic surgery generally requires multiple $(\geq 4)$ skin incisions for robotic arms and patient-side surgeons. In addition, the use of multiple arms results in interreference between the arms and the patient-side surgeon. In the current study, we reviewed our initial experience of a less invasive robotic approach for lung cancer.

Methods: We used 3 arms of the Da Vinci Xi system in an original manner: the camera was set at the most ventral arm for patients and the forceps for right and left hands were set at the more dorsal arms. We made a $4-\mathrm{cm}$ incision in the eighth intercostal space along the middle-axillary line for the insertion of 2 ports for a camera and forceps. This window was eventually used for the extraction of the resected lobes. In addition, we made $1-\mathrm{cm}$ incision along the posterior-axillary line for the remaining arm, and a $1.5-\mathrm{cm}$ incision along the anterior-axillary line for a utility window for the patient-side surgeon.

Results: Our port setting contributed to preventing interference between the 2 adjacent arms (camera and forceps), as well as to improving the performance of the patient-side surgeon who does not experience interference from the robotic arms. During the initial experiences of 39 patients, the same procedure was successfully completed by 3 different console surgeons. There were no catastrophic events during the operations or in the 90-day postoperative period, although we experienced 2 open conversions (5\%) for noncritical bleeding.

Conclusions: We established a 3-incision robotic surgery for lung cancer, which in addition to being patient-friendly, may facilitates collaboration between the console-surgeon and patient-side surgeon without compromising the performance of the console surgeon.
\end{abstract}

Keywords: Lung cancer; robotic surgery; reduced port surgery

Submitted Aug 29, 2021. Accepted for publication Oct 14, 2021.

doi: $10.21037 /$ tcr-21-1772

View this article at: https://dx.doi.org/10.21037/tcr-21-1772

\section{Introduction}

Robotic surgery for lung cancer has become widespread in developed countries. A robotic system enables intuitive handling of the forceps under excellent 3 -dimensional vision. However, regardless of the current trend in reduced port surgery, robotic surgery generally requires multiple ( $\geq 4)$ skin incisions for robotic arms and patientside surgeons (1-4). In addition, the use of multiple arms results in interreference between the arms and the patientside surgeon, particularly during handling the stapler by the patient-side surgeons. We report our initial experience of 3-incision robotic surgery (Figure 1), which enables excellent collaboration between the console surgeon and the patient-side surgeon. We present the following article in accordance with the STROBE reporting checklist (available at https://dx.doi.org/10.21037/tcr-21-1772). 

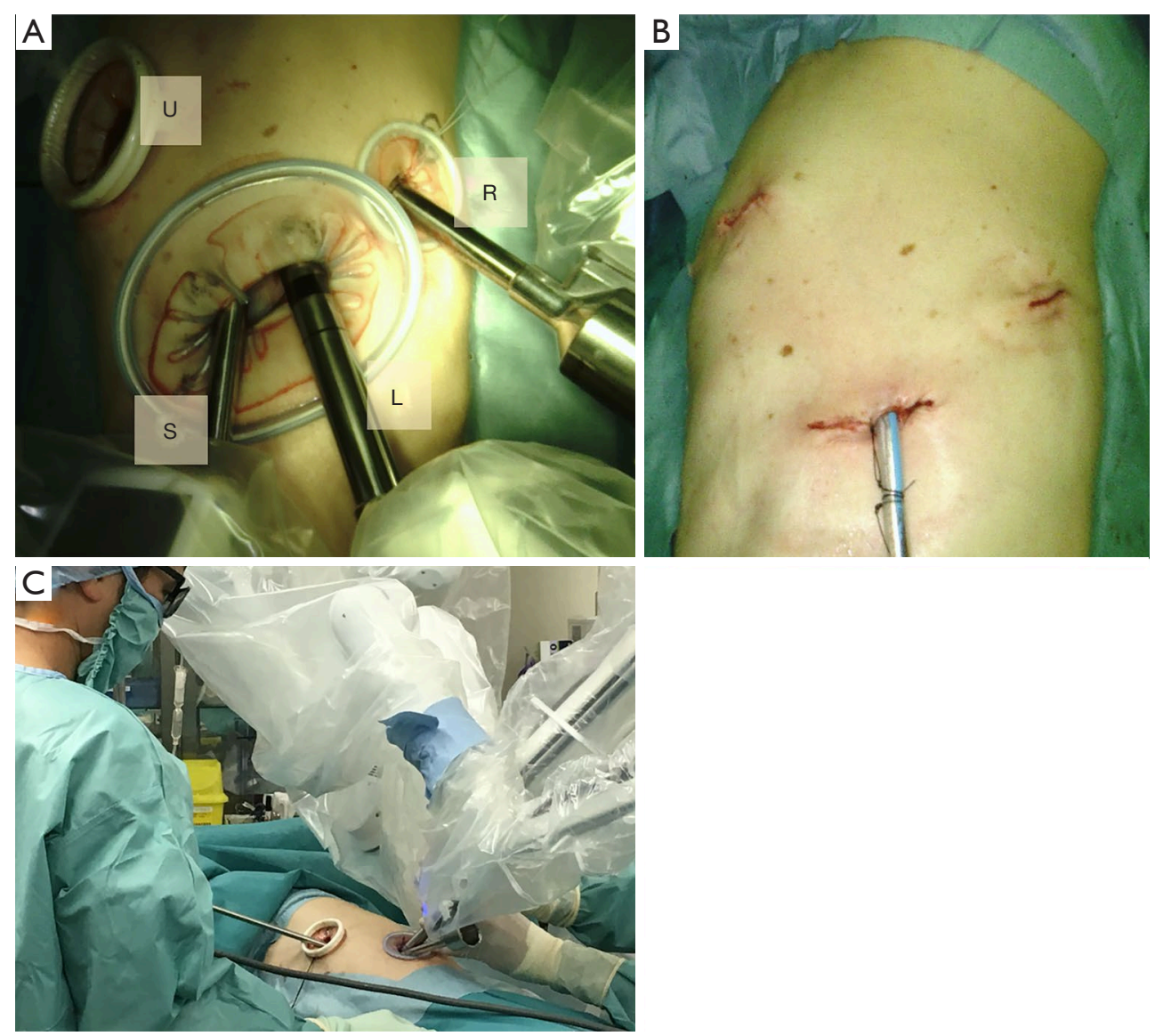

Figure 1 A representative style of 3-incision robotic surgery. Port placement for a left-side operation is shown in (A). A small utility window $(\mathrm{U})$ is placed at the fifth intercostal space. Three da Vinci trocars are placed at the eighth intercostal space. Note that the trocars for the scope $(\mathrm{S})$ and left hand $(\mathrm{L})$ are placed via the same window, and that the trocar for the scope is located at the most ventral side among the 3 da Vinci trocars: the trocar for right hand (R) is placed at the eighth intercostal space. (B) shows 3 wounds with a chest tube. Using our port placement, the patient-side surgeon can freely use the forceps without interference from the robotic arms $(\mathrm{C})$.

\section{Methods}

This was a retrospective analysis of our prospective database of patients who underwent pulmonary resection surgery. This study was conducted in accordance with the Declaration of Helsinki (as revised in 2013). This study was approved by our Institutional Review Board of Kagoshima University Graduate School of Medical and Dental Sciences (No. 210053) and informed consent was obtained from all patients. Before introduction of the 3-incision method, we performed robotic surgery via 4 - or 5 -port using 3 or 4 robotic arms. However, we frequently experienced interference between robotic arms and patient-side surgeon during moving the lung, sucking the blood, and using the stapler or energy devices by the patient-side surgeon $(n=14)$.
We introduced 3-incision robotic surgery for lung cancer in our 15th case undergoing robotic surgery in November 2019. As of February 2021, we had experienced 39 patients undergoing robotic major lung resection for primary lung cancer or metastatic lung cancer. We considered that patients with lung cancer, a solid diameter of $\leq 30 \mathrm{~mm}$, and the absence of lymphadenopathy were eligible for robotic surgery. We collected clinical data, including age, gender, height, weight, smoking history, pulmonary function test results, tumor etiology (primary or metastatic), tumor location, surgical time, console time, blood loss, surgical procedure, length of drainage, open conversion, and postoperative complication. The characteristics of the patients who underwent 3 -incision robotic surgery are shown in Table 1. 
Table 1 Patient characteristics $(n=39)$

\begin{tabular}{lc}
\hline Variables & No. or mean \pm SD \\
\hline Age (years) & $65.4 \pm 10.9$ \\
Gender, male/female & $16 / 23$ \\
Disease, primary/metastatic & $36 / 3$ \\
Height (cm) & $158 \pm 9.3$ \\
Range & $140-178$ \\
BMl (kg/m $\left.{ }^{2}\right)$ & $23.3 \pm 4.0$ \\
Smoking & \\
Yes/No & $21 / 18$ \\
Pack/years & $8.7 \pm 10.0$ \\
\%FVC & $103.6 \pm 15.0$ \\
\%FEV1 & $99.7 \pm 19.9$ \\
FEV /FVC & $0.77 \pm 0.09$ \\
$\% D L C O$ & $100.7 \pm 22.3$ \\
Maximum tumor size (mm) & $22.2 \pm 8.7$ \\
Consolidation tumor size (mm) & $15.4 \pm 7.1$ \\
Tumor location, RU/RM/RL/LU/LL & $15 / 2 / 9 / 10 / 3$ \\
\hline
\end{tabular}

$\mathrm{SD}$, standard deviation; BMI, body mass index; FVC, forced vital capacity; FEV1, forced expiratory volume in 1 second; DLCO, diffusing capacity of carbon monoxide; RU, right upper lobe; $\mathrm{RM}$, right middle lobe; RL, right lower lobe; LU, left upper lobe; LL, left lower lobe.

The 3 arms of the Da Vinci Xi system were used for all cases: No. 1-3 arms were used in the right-side operation and No. 2-4 arms were used in the left-side operation. A $<2$-cm utility window was placed in the fifth intercostal space along the anterior-axillary line for the patientside surgeon (Figure 1A). Thus, the patient-side surgeon stood on the ventral side of the patient. The second $4-\mathrm{cm}$ incision was made in the eighth intercostal space along the middle-axillary line for the insertion of 2 da Vinci trocars for a camera ( $8 \mathrm{~mm}, 30^{\circ}$ angled down scope) and a forceps $(12 \mathrm{~mm})$. This window was eventually used for the extraction of the resected lobes. The third 1-cm incision was made for the insertion of an $8-\mathrm{mm}$ da Vinci trocar along the posterior-axillary line for the remaining arm. The camera was set at the most ventral arm for patients (No. 3 arm in right operation and No. 2 arm in left operation) and the forceps was set at the remaining 2 arms. da Vinci stapler was inserted via $12-\mathrm{mm}$ trocar adjacent to the camera trocar without exception. The da Vinci trocars were inserted via the ninth intercostal space in some patients who underwent lower lobectomy or whose height was $<150 \mathrm{~cm}$. The patient-side surgeon generally used multiple curved forceps, which were designed for single-port thoracoscopic surgery, via a utility incision. If necessary, the patient-side surgeon used endoscopic staplers, vessel sealing devices, and knot sliders for vessel ligation. We did not use a $\mathrm{CO}_{2}$ insufflation system in the current 39 patients.

\section{Statistical analyses}

The values were expressed as the mean \pm standard deviation (SD). An exact $t$-test was used to compare continuous variables between the groups. $\mathrm{P}$ values of $<0.05$ were considered to indicate statistical significance. The statistical analyses were performed using the STATA 12 software program (Stata Corp., College Station, TX, USA).

\section{Additional assessment for pain}

Postoperative pain was assessed when the patients were at rest, $3 \mathrm{~h}$ after the operation (postoperative day- 0 ) and on every postoperative day for the following week (postoperative day 1-7) using a numerical rating scale (NRS). The pain scale ranged from 0 (no pain) to 10 (excruciating pain). The NRSs on each postoperative day in the current 39 patients were compared to those of 14 patients who underwent lobectomy for primary lung cancer before November 2019. The 14 patients underwent robotic surgery via 4 or 5 incisions that were substantially placed at 3 or more intercostal spaces.

\section{Results}

Video 1 provides a summary of the 3 -incision robotic surgery. Thirty-seven patients (95\%) underwent 3-incision robotic surgery and 2 patients $(5 \%)$ underwent open conversion: one patient had silicotic lymph node adhesion to the pulmonary artery and the other patient had accidental pulmonary arterial injury (total blood loss: 350 and $956 \mathrm{~g}$, respectively). The mean operating time was $255 \mathrm{~min}$, the console time was $198 \mathrm{~min}$, blood loss was $145 \mathrm{~mL}$, and the drainage period was 2.3 days (Table 2). During 30-day postoperative period, there were 5 cases with morbidity of any grade (Clavien-Dindo classification) (12.8\%), 3 cases with grade $\geq 3$ morbidity ( $7.7 \%$ ) (Table 2 ), namely wound 


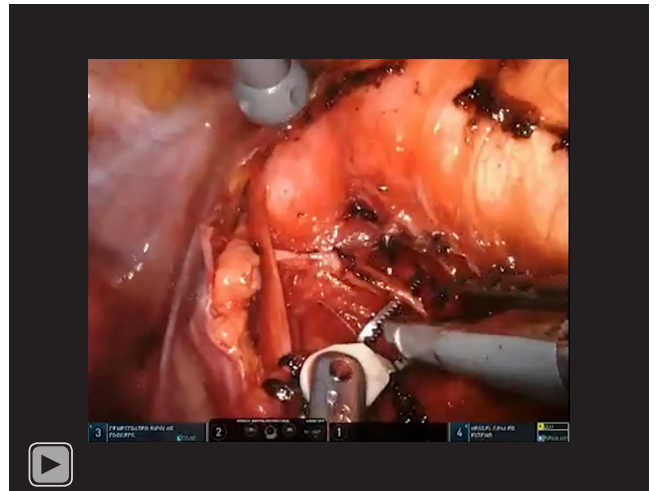

Video 1 The video shows the port placement, arm setting, and surgical procedures in a representative case undergoing en bloc left upper lobectomy for primary lung adenocarcinoma. We used three robotic arms in the left-side surgery (No. 2-4 arms). The scope was set at No. $2 \mathrm{arm}$. This setting brings us wide space at anterior chest wall, facilitating patient-side surgeon in cooperating the surgery. During tracheobronchial lymph node dissection, patientside surgeon effectively moves the left upper lobe toward the caudal direction. Da Vinci stapler is always driven by No. 3 arm in the left-side surgery during cutting the incomplete fissures and pulmonary bronchovasculatures. Note that patient-side surgeon actively assists in cutting the incomplete fissure and creating surgical view during hilar node dissection by pulling the upper lobe and elevating pulmonary artery. The partially mobilized tracheobronchial lymph nodes are pulled caudally between the pulmonary artery and the bronchus, and resected together with the let upper lobe (en bloc left upper lobectomy).

Table 2 Surgical outcomes ( $\mathrm{n}=39$ )

\begin{tabular}{lc}
\hline Variables & No. or mean \pm SD \\
\hline Operation, Lob/Seg & $36 / 3$ \\
Operating time (min) & $255 \pm 71$ \\
Console time (min) & $198 \pm 71$ \\
Length of drainage (days) & $2.3 \pm 2.0$ \\
Blood loss (g) & $145 \pm 200$ \\
Open conversion, Yes/No & $2 / 37$ \\
Postop. complication, Yes/No & $5 / 34$ \\
\hline
\end{tabular}

infection, atelectasis, and reoperation for port-site bleeding. Two patients who underwent open conversion recovered without postoperative complications. There was no 90-day mortality.

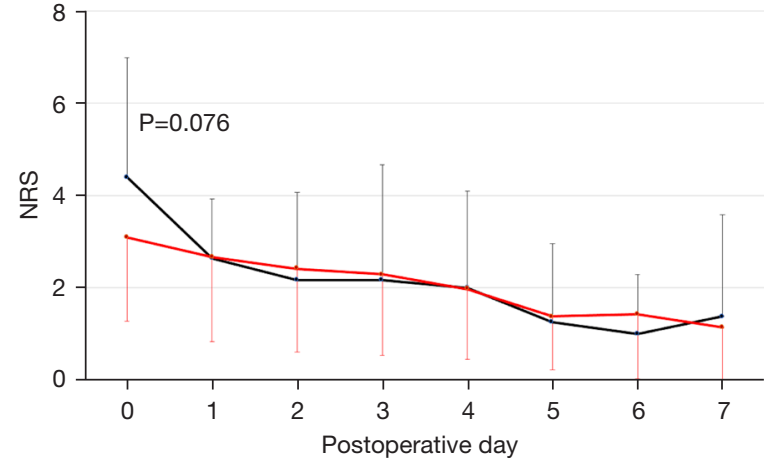

Figure 2 Comparison of the numerical rating scale (NRS) for postoperative pain at rest on each postoperative day among 39 patients undergoing 3 -incision robotic surgery (red) and 14 patients undergoing 4- or 5-incision robotic surgery (black). The mean NRS of the 14 patients undergoing 4- or 5 -incision robotic surgery on the day of surgery was slightly higher than that of the current 39 patients $(\mathrm{P}=0.076)$.

Console surgeons basically used 2 forceps, a Fenestrated bipolar forceps and a Maryland bipolar forceps, except for the additional use of curved monopolar scissors in the initial six cases (mean, 2.2 forceps per case). A vessel sealing device was used either by the console surgeon $(n=7)$ or by the patient-side surgeon $(n=31)$. A stapling device was basically used by the console surgeon, but that was used by the patient-side surgeon during the stapling of the lobar bronchus in four patients: the mean number of cartridges used per patient was 6 .

The operation was performed by any of 3 console surgeons. There was no apparent learning curve with regard to console time. Rather, the console time was surgeon dependent: the console time was $245 \pm 64 \mathrm{~min}$ for console surgeon A $(n=18), 167 \pm 41 \mathrm{~min}$ for console surgeon B $(n=16)$, and $160 \pm 44 \mathrm{~min}$ for console surgeon $C(n=5)$. The console time of console surgeon A was significantly longer in comparison to console surgeon $\mathrm{B}(\mathrm{P}<0.001)$ or console surgeon $\mathrm{C}(\mathrm{P}=0.013)$.

With regard to postoperative pain, the mean NRS for pain in the current 39 patients who underwent 3 -incision robotic surgery was below 4 at each time point (Figure 2). In contrast, the mean NRS for pain in the previous 14 patients who underwent 4 - or 5-incision robotic surgery was $>4$ on the day of surgery, which was marginally higher than that of the current 39 patients $(\mathrm{P}=0.076)$. None of the 53 patients complained of chronic neuropathic pain which required some analgesics beyond postoperative day 30 . 
A

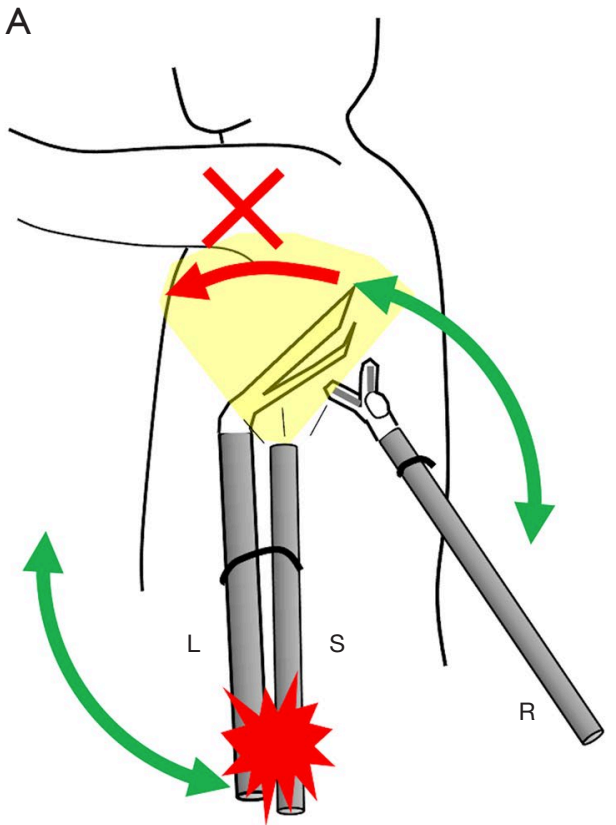

B

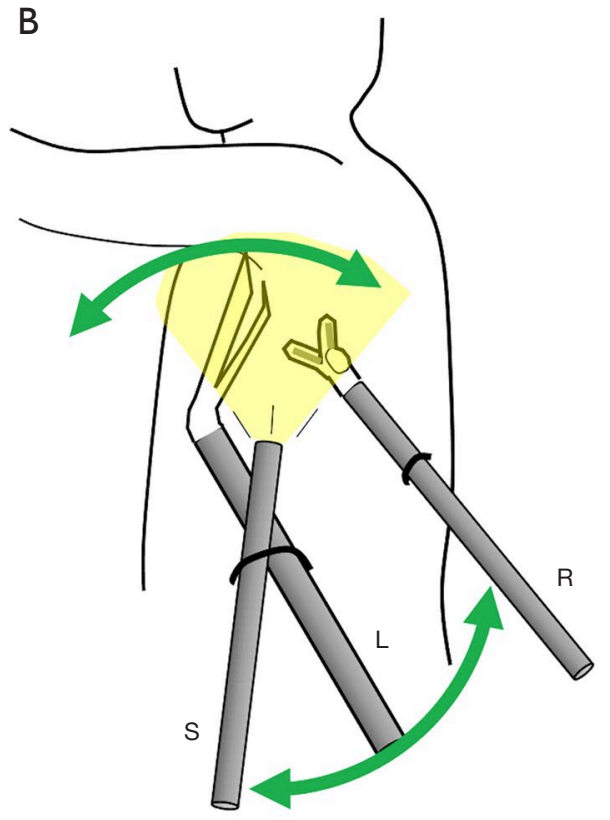

Figure 3 Comparison of two different instrumentations in 3-incision approach (A: co-axial method; B: para-axial method). (A) shows the 3 -incision approach by placing the camera port (S) between the arms for the right hand (R) and left hand (L); the so-called co-axial method. This arm setting results in interference between the arm for the camera and the adjacent arm for the forceps, thereby limiting the range of motion of the forceps. In contrast, the current method, the so-called para-axial method, was revealed to be useful in preventing interference between the arms (B).

\section{Discussion}

Although thoracoscopic surgery for lung cancer has become popular worldwide, not all surgeons are familiar with thoracoscopic surgery-in particular singleincision thoracoscopic surgery-probably because of the considerable gap between the motion of the hands and forceps in comparison to open thoracotomy under direct vision. Robotic surgery has an advantage with regard to ergonomics, which facilitates intuitive handling of the forceps during port access surgery. However, conventional robotic surgery requires at least four skin incisions (usually 5 or 6 incisions). In the current report, we introduced a novel, 3 -incision robotic surgery procedure for lung cancer (Figure 1B). According to our initial experiences, our methods were revealed to be reproducible: three surgeons successfully completed the 3 -incision surgical procedures with acceptable early outcomes. In addition, our method was applicable to small or obese patients: nine patients were $<150 \mathrm{~cm}$ in height and 3 patients had a body mass index of $>30 \mathrm{~kg} / \mathrm{m}^{2}$. We believe that our 3 -incision approach is a valid method of minimally invasive robotic surgery for lung cancer.

Before introducing the current 3 -incision robotic surgery procedure, we attempted a 3 -incision approach with placement of the camera port between the arms for the right and left hands; the so-called co-axial method (Figure 3A). Unfortunately, the arm for camera and the adjacent arm for the forceps interfered with each other, resulting in a limitation in the movable range of the forceps (Figure 3A). In contrast, the current method, the so-called para-axial method, was found to be useful for preventing interference between the arms (Figure 3B). As we described above, any bronchus, pulmonary vessels, and incomplete fissures could be dissected by robotic staplers that were inserted via trocars adjacent to the camera: we never used another arm for the stapler.

One may consider that our method may be uncomfortable or may prevent the intuitive handling of forceps. However, as can be seen in the videoclip, the procedure appears natural in the magnified view, as if the surgeon is performing surgery via a co-axial view. In addition, the console surgeon can comfortably perform surgery because of the excellent collaboration between the 
console surgeon and the patient-side surgeon (5), since all of the robotic arms are located behind the middle-axillary line, the patient-side surgeon can freely assist surgery via the utility window placed along the anterior-axillary line without interference from the robotic arms (Figure 1C). We are also familiar with single-port thoracoscopic lung lobectomy which is performed via a small window, similarly to the current utility window for the patient-side surgeon. We used multiple curved forceps and a curved suction device via the utility window in the current robotic surgery, similarly to single-port surgery. Such valuable assistance from the patient-side surgeon enabled comfortable surgery via excellent vision without using $\mathrm{CO}_{2}$ insufflation.

With regard to postoperative pain, we mainly used intercostal nerve block based on the results of previous studies $(6,7)$. Local analgesics were administered just before chest wall closure, mainly to the fifth- and eighthintercostal space, where the utility window and da Vinci trocar were placed, respectively. Because the chest wall damage was limited to the 2 intercostal spaces, our robotic approach may be advantageous in minimizing postoperative pain: the postoperative pain of the current 39 patients appeared to be less severe than that of the previous 14 patients in whom the chest wall damage was distributed to 3 or more intercostal spaces, although the difference was not statistically significant, probably because of the limited sample size. In addition to the acute postoperative pain, it was suggested that the 3 -incision method did not enhance the chronic postoperative pain, including neuropathic pain, which required some analgesics.

Robotic surgery is generally more costly than thoracoscopic surgery because of higher expenditure for consumable items, such as robotic forceps and additional items for $\mathrm{CO}_{2}$ insufflation (expenditure for staplers and vessel sealing devices are comparable between robotic surgery and thoracoscopic surgery) $(8,9)$. In the current patients, we basically used only 2 forceps per patient without $\mathrm{CO}_{2}$ insufflation. Therefore, we believe that our method is not only minimally invasive, but also minimizes the cost of robotic lobectomy for cancer.

There is a potential limitation in this study: this was a retrospective one-arm study showing our initial experience of three-incision robotic surgery. In addition, although postoperative pain was compared to historical control, the small sample size could compromise reliable statistical analysis. Although the 3 -incision robotic surgery was successfully accomplished by three authors in this study, multicenter study with more sample size is needed before the 3 -incision robotic surgery achieves as a reliable minimally incision robotic surgery.

In conclusion, we established a 3-incision robotic surgery procedure for lung cancer that is patient-friendly and which also facilitates collaboration between the console-surgeon and patient-side surgeon without compromising the performance of the console surgeon.

\section{Acknowledgments}

Funding: None.

\section{Footnote}

Reporting Checklist: The authors have completed the STROBE reporting checklist. Available at https://dx.doi. org/10.21037/tcr-21-1772

Data Sharing Statement: Available at https://dx.doi. org/10.21037/tcr-21-1772

Peer Review File: Available at https://dx.doi.org/10.21037/ tcr-21-1772

Conflicts of Interest: All authors have completed the ICMJE uniform disclosure form (available at https://dx.doi. org/10.21037/tcr-21-1772). The authors have no conflicts of interest to declare.

Ethical Statement: The authors are accountable for all aspects of the work in ensuring that questions related to the accuracy or integrity of any part of the work are appropriately investigated and resolved. This study was conducted in accordance with the Declaration of Helsinki (as revised in 2013). The study was approved by our Institutional Review Board of Kagoshima University Graduate School of Medical and Dental Sciences (No. 210053) and informed consent was taken from all the patients.

Open Access Statement: This is an Open Access article distributed in accordance with the Creative Commons Attribution-NonCommercial-NoDerivs 4.0 International License (CC BY-NC-ND 4.0), which permits the noncommercial replication and distribution of the article with the strict proviso that no changes or edits are made and the original work is properly cited (including links to both the formal publication through the relevant DOI and the license). 
See: https://creativecommons.org/licenses/by-nc-nd/4.0/.

\section{References}

1. 1. Melfi FM, Menconi GF, Mariani AM, et al. Early experience with robotic technology for thoracoscopic surgery. Eur J Cardiothorac Surg 2002;21:864-8.

2. Rajaram R, Mohanty S, Bentrem DJ, et al. Nationwide Assessment of Robotic Lobectomy for Non-Small Cell Lung Cancer. Ann Thorac Surg 2017;103:1092-100.

3. Huang J, Luo Q, Tan Q, et al. Initial experience of robotassisted thoracoscopic surgery in China. Int J Med Robot 2014;10:404-9.

4. Kent M, Wang T, Whyte R, et al. Open, video-assisted thoracic surgery, and robotic lobectomy: review of a national database. Ann Thorac Surg 2014;97:236-42; discussion 242-4.

5. Sgarbura O, Vasilescu C. The decisive role of the

Cite this article as: Ueda K, Umehara T, Maeda K, Suzuki S, Yokomakura N, Kariatsumari K, Sato M. Three-incision robotic major lung resection for cancer. Transl Cancer Res 2021;10(11):4617-4623. doi: 10.21037/tcr-21-1772 patient-side surgeon in robotic surgery. Surg Endosc 2010;24:3149-55.

6. Bolotin G, Lazarovici H, Uretzky G, et al. The efficacy of intraoperative internal intercostal nerve block during video-assisted thoracic surgery on postoperative pain. Ann Thorac Surg 2000; 70:1872-5.

7. Ueda K, Hayashi M, Murakami J, et al. Intercostal block vs. epidural analgesia in thoracoscopic lung cancer surgery: a randomized trial. Gen Thorac Cardiovasc Surg 2020;68:254-60.

8. Augustin F, Bodner J, Maier H, et al. Robotic-assisted minimally invasive vs. thoracoscopic lung lobectomy: comparison of perioperative results in a learning curve setting. Langenbecks Arch Surg 2013;398:895-901.

9. Nakamura H, Suda T, Ikeda N, et al. Initial results of robot-assisted thoracoscopic surgery in Japan. Gen Thorac Cardiovasc Surg 2014;62:720-5. 